\title{
Differential Response of Period 1 Expression within the Suprachiasmatic Nucleus
}

\author{
Wataru Nakamura, Shin Yamazaki, Nana N. Takasu, Kazuo Mishima, and Gene D. Block \\ Center for Biological Timing and Department of Biology, University of Virginia, Charlottesville, Virginia 22903-2477
}

\begin{abstract}
The suprachiasmatic nuclei ( $\mathrm{SCNs}$ ) of the hypothalamus contain a circadian clock that exerts profound control over rhythmic physiology and behavior. The clock consists of multiple autonomous cellular pacemakers distributed throughout the rat SCN. In response to a shift in the light schedule, the SCN rapidly changes phase to achieve the appropriate phase relationship with the shifted light schedule. Through use of a transgenic rat in which rhythmicity in transcription of the Period 1 gene was measured with a luciferase reporter (Per1-luc), we have been successful in tracking the time course of molecular rhythm phase readjustments in different regions of the SCN that occur in response to a shift in the light schedule. We find that different regions of the SCN phase adjust at different rates, leading to transient internal desynchrony in Per1-luc expression among SCN regions. This desynchrony among regions is most pronounced and prolonged when the light schedule is advanced compared with light schedule delays. A similar asymmetry in the speed of phase resetting is observed with locomotor behavior, suggesting that phase shifting kinetics within the SCN may underlay the differences observed in behavioral resetting to advances or delays in the light schedule.
\end{abstract}

Key words: circadian; desynchronization; entrainment; light; suprachiasmatic; synchrony

\section{Introduction}

In mammals, the principal circadian pacemaker driving daily behavioral rhythms is located in the suprachiasmatic nucleus (SCN) in the anterior hypothalamus (Moore and Eichler, 1972; Stephan and Zucker, 1972; Ralph et al., 1990). The recent identification of mammalian clock genes has revealed that in addition to the SCN, other brain regions and non-neural tissues exhibit circadian oscillations in molecular cycling over which the SCN appears to act as a central pacemaker (Balsalobre et al., 1998; Yamazaki et al., 2000; Abe et al., 2002; Reppert and Weaver, 2002; Yoo et al., 2004). The retinohypothalamic tract conveys photic information to the SCN, entraining the circadian pacemaker to the $24 \mathrm{~h}$ environmental cycle (Moore, 1973; Daan and Pittendrigh, 1976). Photic cues are the most important entraining signals, and it is well established that the greatest density of retinal fibers travel to an area of the SCN referred to as the ventrolateral region and are more sparse in the area called the dorsomedial

Received July 11, 2004; revised April 23, 2005; accepted April 27, 2005.

This work was supported by National Institute of Mental Health Grant MH62517. We thank Hajime Tei for generating the Per1-luc rats, M. E. Geusz for technical information about luminescence imaging, and Johanna Meijer for comments on a previous draft of this manuscript.

Correspondence should be addressed to Gene D. Block, Department of Biology, University of Virginia, P.0. Box 400226, Charlottesville, VA 22904-4226. E-mail: gdb@virginia.edu.

W. Nakamura's present address: Department of Oral Functional Science, Hokkaido University Graduate School of Dentistry, Sapporo 060-8586, Japan.

S. Yamazaki's present address: Department of Biological Sciences, Vanderbilt University, VU Station B, Box 35-1634, Nashville, TN 37235-1634.

N. N. Takasu's present address: Department of Physiology, Hokkaido University Graduate School of Medicine, Sapporo 060-8638, Japan.

K. Mishima's present address: Department of Neuropsychiatry, Akita University School of Medicine, Akita City, Akita 010-8543, Japan.

D01:10.1523/JNEUROSCI.0889-05.2005

Copyright $\odot 2005$ Society for Neuroscience $\quad$ 0270-6474/05/255481-07\$15.00/0 region (Johnson et al., 1988; Moga and Moore, 1997; Muscat et al., 2003). These studies and others indicate that SCN cells are neither functionally nor regionally homogeneous (Hamada et al., 2001; Nakamura et al., 2001; Quintero et al., 2003; Schaap et al., 2003; Yamaguchi et al., 2003).

How light entrains the SCN clock is not fully understood. The clock genes Period 1 (Per1) and Per2, which exhibit circadian expression within the SCN, appear to play a key role in lightinduced resetting of the mammalian clock, insomuch as both genes are rapidly induced after light stimulation at night (Albrecht et al., 1997; Shearman et al., 1997; Shigeyoshi et al., 1997; Tei et al., 1997). Induction of Perl is required for light/glutamateinduced phase shifts in both early (Akiyama et al., 1999) and late night (Tischkau et al., 2003). After a shift in the light schedule, circadian rhythms reentrain to the new light/dark (LD) cycle, although a complete readjustment can take several cycles, especially for phase advances. During the readjustment period, desynchronization appears to develop among different oscillatory tissues and brain regions that readjust their phases at different rates (Yamazaki et al., 2000; Stokkan et al., 2001; Abe et al., 2002; Nagano et al., 2003). In previous studies on reentrainment to phase-advanced light schedules, it was shown that the circadian rhythm of Perl expression in the entire SCN shifted more rapidly than did the rhythm of locomotor behavior, Perl expression in peripheral tissues (Yamazaki et al., 2000), or SCN electrical activity measured in vivo or in vitro (Vansteensel et al., 2003).

The present study was performed in an effort to explore the resetting kinetics of different region of the rat SCN using realtime imaging of Per1-luciferase (Per1-luc) activity in a Per1luciferase transgenic rat model developed by Hida et al. (2000) and Yamazaki et al. (2000). This rat model allows us to continu- 
ously observe the time course of Per1-luc rhythmicity, in vitro, in all regions of the SCN.

\section{Materials and Methods}

Animals. Perl-luciferase transgenic rat line $\mathrm{W}$ (perl)2, originally generated by Hida et al. (2000) and Yamazaki et al. (2000), was used in these experiments, using the same strategy as in previous studies. The $\mathrm{W}$ (perl)2 rats (homozygous male; at 7-9 weeks of age) exhibited a freerunning period in constant darkness, $24.48 \pm$ $0.03 \mathrm{~h}(\mathrm{SE} ; n=12)$, which is very close to the period of the $\mathrm{W}$ (perl) 1 heterozygote $(24.43 \pm$ $0.02 \mathrm{~h} ; n=20$ ) (Yamazaki et al., 2000) and wild types $(24.33 \pm 0.01)$. There are $\sim 50$ copies per genome of the transgene integrated in $\mathrm{W}$ (perl)2. Male homozygous W(perl)2 (1-4 months of age), which displayed brighter luminescence than $\mathrm{W}$ (perl) 1 in the SCN, were used for this study. All rats were raised in the animal facility under a $12 \mathrm{~h} \mathrm{LD}$ cycle (lights on at 5:00 A.M., lights off at 5:00 P.M.). Lights off was defined as Zeitgeber time (ZT) 12 . The $6 \mathrm{~h}$ advance in the light schedule was accomplished by advancing the time of lights on, leading to one short $6 \mathrm{~h}$ night. The $6 \mathrm{~h}$ delay was accomplished by delaying the time of lights off, resulting in one long $18 \mathrm{~h}$ day. The light cycle continued after the light schedule advance or delay. Rats were killed just before lights off after their first, third, or sixth (for phase advance only) complete light cycle. Light intensity was $30-60 \mu \mathrm{W} /$ $\mathrm{cm}^{2}$ (40 W fluorescent lamp; 100-200 lux at cage level; Philips, Somerset, NJ). Food and water were available ad libitum throughout the experiments. All experiments were performed under the approval of the Committee on Animal Care and Use at the University of Virginia.

Per1-luc bioluminescence. Within $30 \mathrm{~min}$ before ZT 12, rats were anesthetized with halothane and decapitated. The brain was removed and placed in cold HBSS. The hypothalamic slice containing paired SCNs $(1.6 \times 2.0 \mathrm{~mm}$; dissected size $)$ was cut from $300 \mu \mathrm{m}$ coronal brain slices made with a vibratome slicer and placed on a culture membrane (Millicell-CM, PICM30-50m; Millipore, Bedford, MA) in $1.2 \mathrm{ml}$ of DMEM (D2902; Sigma, St. Louis, MO) supplemented with 2\% B27 (Invitrogen, San Diego, CA), 10 mM HEPES, 25,000 U/L penicillin, 25,000 $\mu \mathrm{g} / \mathrm{L}$ streptomycin, $352.5 \mathrm{mg} / \mathrm{L}$ sodium bicarbonate, $3.5 \mathrm{~g} / \mathrm{L}$ D-glucose, and $0.1 \mathrm{~mm}$ luciferin (Promega, Madison, WI). These SCN slices were sealed in a $35 \mathrm{~mm}$ Petri dish with a coverslip and vacuum grease and maintained at $36^{\circ} \mathrm{C}$ in darkness.

Bioluminescence from the SCN slices was measured with an intensified charge-coupled device (ICCD) camera (C2400) and an Argus-50 photon-counting image processor from Hamamatsu Photonic Systems (Hamamatsu, Japan). SCN slices were imaged from above with tandemmounted $50 \mathrm{~mm}$ f/1.2 Nikon (Tokyo, Japan) camera lenses attached to the ICCD camera. The modules and cultures were enclosed in a lighttight chamber (Hamamatsu Photonic Systems) inside a temperaturecontrolled incubator at $36^{\circ} \mathrm{C}$ and interfaced to image processor for continuous data acquisition. Photon counts were integrated $25 \mathrm{~min}$ at every 30 min intervals.

Quantitative analysis. The outer border of the SCN was defined by the image at the peak phase. We created a template of the SCN and analyzed the luminescence of serial images. Unilateral SCNs were divided into 15 regions, each region containing $5 \times 5$ pixels. Perl-luc luminescence was quantified using the Scion (Frederick, MD) Image program (version Beta 4.0.2) and was represented as the mean gray value per pixel in an eight-bit gradient. The peaks in each section of the SCN were determined by using a five-point $(2.5 \mathrm{~h})$ moving average time window. The highest point during the first complete cycle in vitro was defined as the peak. Two-way ANOVA was performed with light schedule (control and $6 \mathrm{~h}$ delays or advances) and sections in the SCN as factors to analyze the phase shift. One-way ANOVA followed by Tukey's test was used to analyze the difference of peak time in each section of the SCN. A section-by-section analysis was performed for both peak times and for trough times.

\section{Results}

\section{Per1-luc rhythms within the SCN}

Brain slices containing the SCN exhibited robust rhythms in Per1-luc luminescence (Fig. 1a). After the start of culture, the luminescence gradually decreased and then increased to show a peak in expression during the projected daytime of the previous light cycle. At the peak, the outer border of the SCN was easily distinguished by virtue of its higher luminescence than the surrounding tissue. We typically obtained three SCN slices in which the first slice contained the anterior part of the SCN and last slice the posterior side. We used the middle slice and applied a template that subdivided each SCN into 15 regions for analysis (Fig. 1b).

We observed that the timing of Perl-luc expression was not uniform across the SCN. Initial increases in the Per1-luc signal first appeared in the dorsomedial SCN around ZT 2. Over time, the Per1-luc signal moved more laterally into the central part of 
Zeitgeber Time (h)

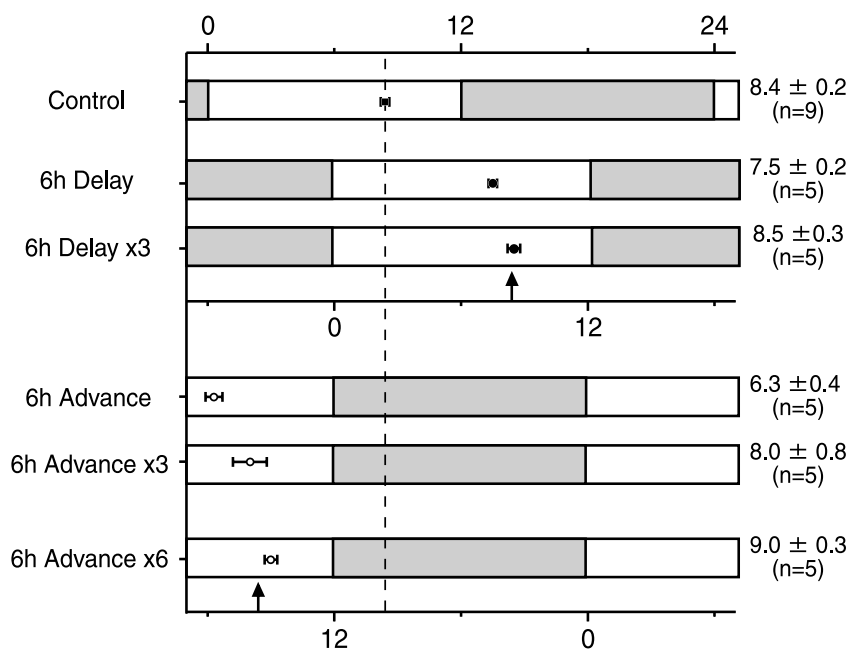

Figure 2. Peak phase of Per1-luc rhythm after shifts in the light schedule. The peak phase represents the averaged luminescence data from the entire $\mathrm{SCN}$. Each bar represents the light schedule before (control) and after a $6 \mathrm{~h}$ phase delay or phase advance. The time of darkness is indicated by the filled bar, and light time is indicated by the open bar. Mean phase $\pm S E$ is indicated to the right of the figure. Vertical arrows indicate the projected steady-state phase for a $6 \mathrm{~h}$ delay or advance in the light schedule.

the SCN toward the ventrolateral region (Fig. 1a). We observed a peak time of ZT $7.8 \pm 0.2$ (mean $\pm \mathrm{SE} ; n=18$ ) for the dorsomedial SCN and ZT $8.6 \pm 0.1$ for the ventrolateral SCN (see Figs. $3 a$, $4 a)$. There was a significant difference in the peak times within the SCN (ANOVA; $\left.F_{(14,238)}=3.91 ; p<0.001\right)$; the dorsomedial region was advanced with respect to ventrolateral areas (Tukey's test; $p<0.05)$. The steady-state phase difference between the dorsomedial and ventrolateral regions appears robust and not dependent on the phase marker used for the measurement. Analysis of phase differences between rhythm troughs revealed a similar result with a mean $1.1 \mathrm{~h}$ difference between the dorsomedial $($ ZT $22.7 \pm 0.3)$ and ventrolateral (ZT 23.8 \pm 0.3$)$ regions.

\section{Per1-luc rhythms after $6 \mathrm{~h}$ phase delays or advances}

To compare our results to a previous reentrainment study (Yamazaki et al., 2000) that used a different transgenic rat line [W(perl) 1 heterozygotes], we measured the time course of phase adjustments of the SCN to $6 \mathrm{~h}$ delays or advances in the light schedule (Fig. 2). Measuring luminescent signals from the entire $\mathrm{SCN}$, as reported previously, we found that phase delays occurred rapidly, with a nearly full adjustment after one cycle and a completed delay by the third light cycle. We also observed rapid phase advances, but our results differed from the previous study (Yamazaki et al., 2000) in that the phase advance was initially larger than $6 \mathrm{~h}$, retarding to the appropriate steady-state advance over the next several days. Thus, although both phase advances and delays occurred rapidly, phase advances initially appeared to "overshoot" and then retarded to the appropriate steady-state value.

\section{Regional Per1-luc rhythms after $6 \mathrm{~h}$ phase delay}

By analyzing the phases of individual regions within the SCN, we observed that the SCN undergoes internal desynchronization during phase adjustments to shifted light schedules. The transient desynchrony involves several regions of the SCN, and the composite behavior of the SCN can best be visualized in a threedimensional representation of phase plotted with respect to po-
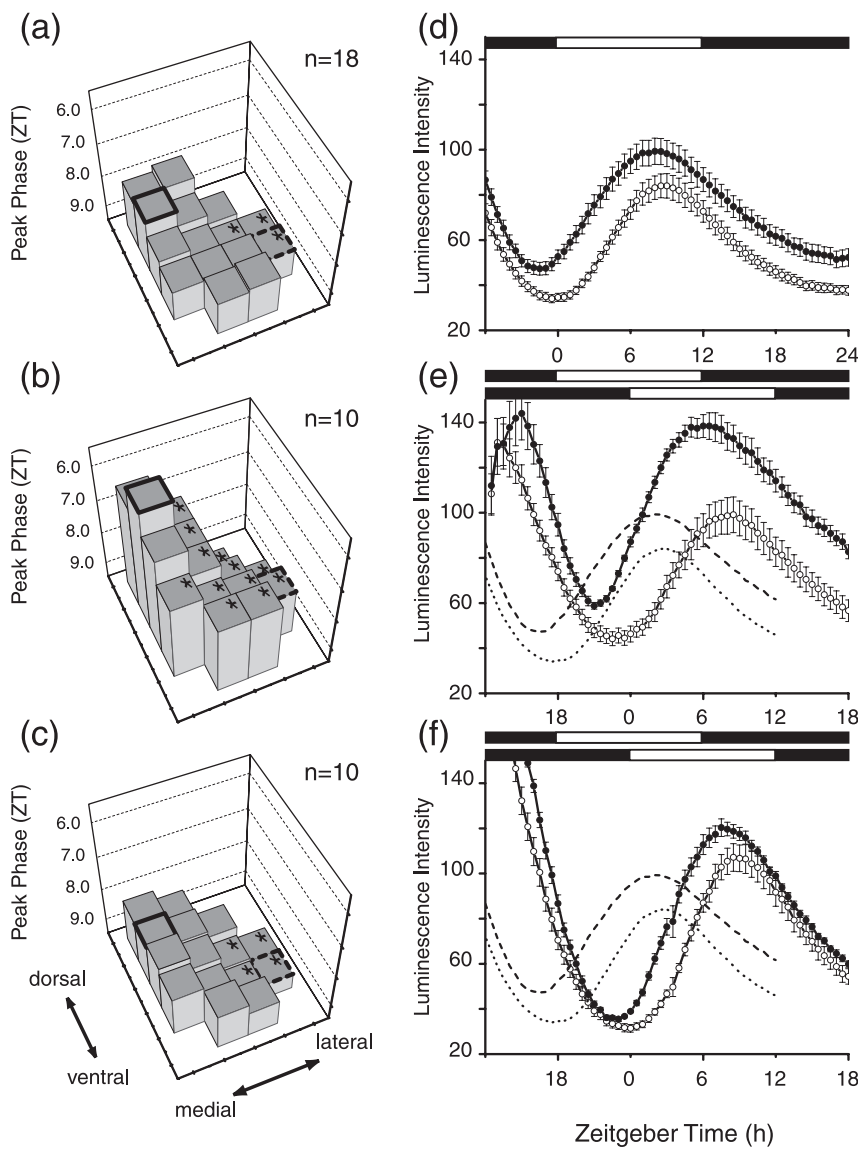

Figure 3. Reentrainment of Per1 expression in the SCN to $6 \mathrm{~h}$ phase delay in the light cycle. $\boldsymbol{a}$, Steady-state phases of the Per1-luc rhythm. The phase of the 15 regions of the SCN are displayed as histograms with the position on the surface of the SCN indicated. For quantitative and statistical comparisons, the bold histogram represents the dorsomedial region, and the histogram surrounded by dashes indicates the ventrolateral histogram. Asterisks on the tops of histograms indicate regions that were statistically significantly different in phase from the dorsomedial region (Tukey's test; $p<0.05$ ). The phase plane formed by the tops of the histograms is tipped upward in the top left corner, revealing that the dorsomedial region phase leads other regions. $\boldsymbol{b}$, Phase plane on day 1 after $6 \mathrm{~h}$ delay in light schedule. The orientation of the plane reveals that the lateral region of the SCN has shifted more than the medial regions. c, By day 3 after the light schedule delay, the phase plane orientation is similar to the control orientation, indicating that the phase shift is essentially complete in all regions of the SCN. $\boldsymbol{d}$, Circadian rhythms in Per1-Iuc expression in dorsomedial (filled circles) and ventrolateral (open circles) regions of the SCN before shift in light schedule (control). $\boldsymbol{e}$, Per 1-luc rhythms on day 1 after light cycle delay. $f$, Per1-luc rhythms on day 3. Controls are shown in dashed lines, dark dashes for dorsomedial region. In $\boldsymbol{d}-\boldsymbol{f}$, error bars indicate SE. ZT is time of light cycle just before recording; thus, ZT is for $6 \mathrm{~h}$ delayed light schedule in $\boldsymbol{b}$ and $\boldsymbol{c}$.

sition within the SCN. Figure 3a displays the steady-state phase relationships of Per1 rhythmicity within the SCN. The tops of the individual histograms form a "phase plane" that is tilted upward in the left corner, indicating that in steady state, the dorsomedial portion of the SCN phase leads other regions, including the ventrolateral region (Fig. 3a). Significant phase difference between the dorsomedial and other regions are indicated by asterisks on each histogram. On the first cycle after a $6 \mathrm{~h}$ phase delay, the phase plane changes orientation, becoming steeper, because of the fact that the ventrolateral and dorsolateral regions of the SCN have delayed more than the dorsomedial and ventromedial regions (Fig. $3 b$ ). By day 3 after the light schedule shift, the phase plane has almost resumed the preshift orientation, indicating that the steady-state phase relationships among the various regions of the SCN have been nearly reestablished (Fig. 3c). An identical analysis using rhythm troughs yielded similar results (data not shown). 
Comparing just the dorsomedial and ventrolateral regions (Fig. 3e), after one complete cycle of $6 \mathrm{~h}$ phase delay, Per 1 expression appeared in the dorsomedial SCN $\sim 11-12 \mathrm{~h}$ after dusk (ZT 23 to ZT 24) and showed peak expression at ZT $5.8 \pm 0.4(n=$ $10)$. The ventrolateral SCN peaked at ZT $8.1 \pm 0.2$. Compared with control (Fig. 3a,d), the dorsomedial SCN showed a $4.0 \mathrm{~h}$ phase delay and the ventrolateral SCN a $5.5 \mathrm{~h}$ phase delay. After three cycles of the $6 \mathrm{~h}$ delayed light schedule, the dorsomedial and ventrolateral regions regained their normal phase relationship (Fig. $3 f$ ). There was significant difference in the peak time within the SCN (ANOVA; $F_{(14,126)}=6.55 ; p<0.001$ ) when compared with control slices (two-way ANOVA; $F_{(2,35)}=547 ; p<0.001$ ). The time required to reestablish the steady-state phase relationships within the SCN was consistent with the time required for reentrainment of wheel-running activity after a $6 \mathrm{~h}$ phase delay (Yamazaki et al., 2000). We also observed what appears to be an increase in the amplitude of luminescence in the dorsomedial region after the phase delay (Fig. $3 e$ ). Although we observed a consistent small difference in peak luminescence between the ventrolateral and dorsomedial regions (Figs. $1 c, 3 a, 4 a$ ), the difference appeared to increase transiently during delaying phase adjustments.

\section{Per1-luc rhythms after $6 \mathrm{~h}$ phase advance}

Phase advances in the light schedule lead to more prolonged desynchronization within the SCN than occurs with delays. On the first cycle after the phase advance, the ventrolateral region shifted significantly more than the dorsomedial and ventromedial regions, leading to an inversion in their normal phase relationship (Fig. 4b). After $3 \mathrm{~d}$ of the new light cycle, the phase relationships among the different regions were not fully restored (Fig. $4 c$ ). Only after $6 \mathrm{~d}$ of the new light regimen were the phase relationships within the SCN fairly well restored to their preshift condition (Fig. 4d). An analysis using rhythm troughs yielded similar results with no significant phase differences among SCN regions on day 3 and the expected phase differences evident by day 6 (data not shown).

Examining the behavior of the dorsomedial and ventrolateral regions, on the first day after the light schedule shift, the dorsomedial SCN showed a broad peak and reversed phase relationship to the ventrolateral SCN compared with unshifted control slices (Fig. $4 e, f$ ). The peak phase in the dorsomedial SCN was ZT 7.9 \pm $0.5(n=10)$ and in the ventrolateral SCN was ZT $5.2 \pm 0.3$. The peak of the ventrolateral SCN at ZT 5.2 is phase advanced by $>9$ $\mathrm{h}$ compared with controls, and thus this region of the SCN appears to have significantly "overshot" the $6 \mathrm{~h}$ phase advance in the light schedule. On day 3, the peak phase in the dorsomedial SCN was ZT $7.9 \pm 0.4(n=10)$ and in the ventrolateral SCN was ZT $8.4 \pm 0.6$ (Fig. $4 c, g$ ). There were no significant differences in the peak times within the SCN on day $3\left(\right.$ ANOVA; $F_{(14,126)}=1.37$; $p=0.18$ ), indicating that the normal steady-state phase differences between the dorsomedial and ventrolateral regions had not yet been reestablished. After six cycles after the phase advance in the light schedule, the rhythm of Perl expression had fully reentrained in the SCN (Fig. 4d,h). The dorsomedial SCN (ZT $8.4 \pm$ $0.2 ; n=10)$ and the ventrolateral SCN (ZT 9.4 \pm 0.3 ) had reestablished their normal phase relationship. As with phase delays, the time taken reestablishing steady-state phase relationships within the SCN after a phase advance appeared to match the time course of behavioral adjustments, with phase advances taking longer than phase delays (Yamazaki et al., 2000).
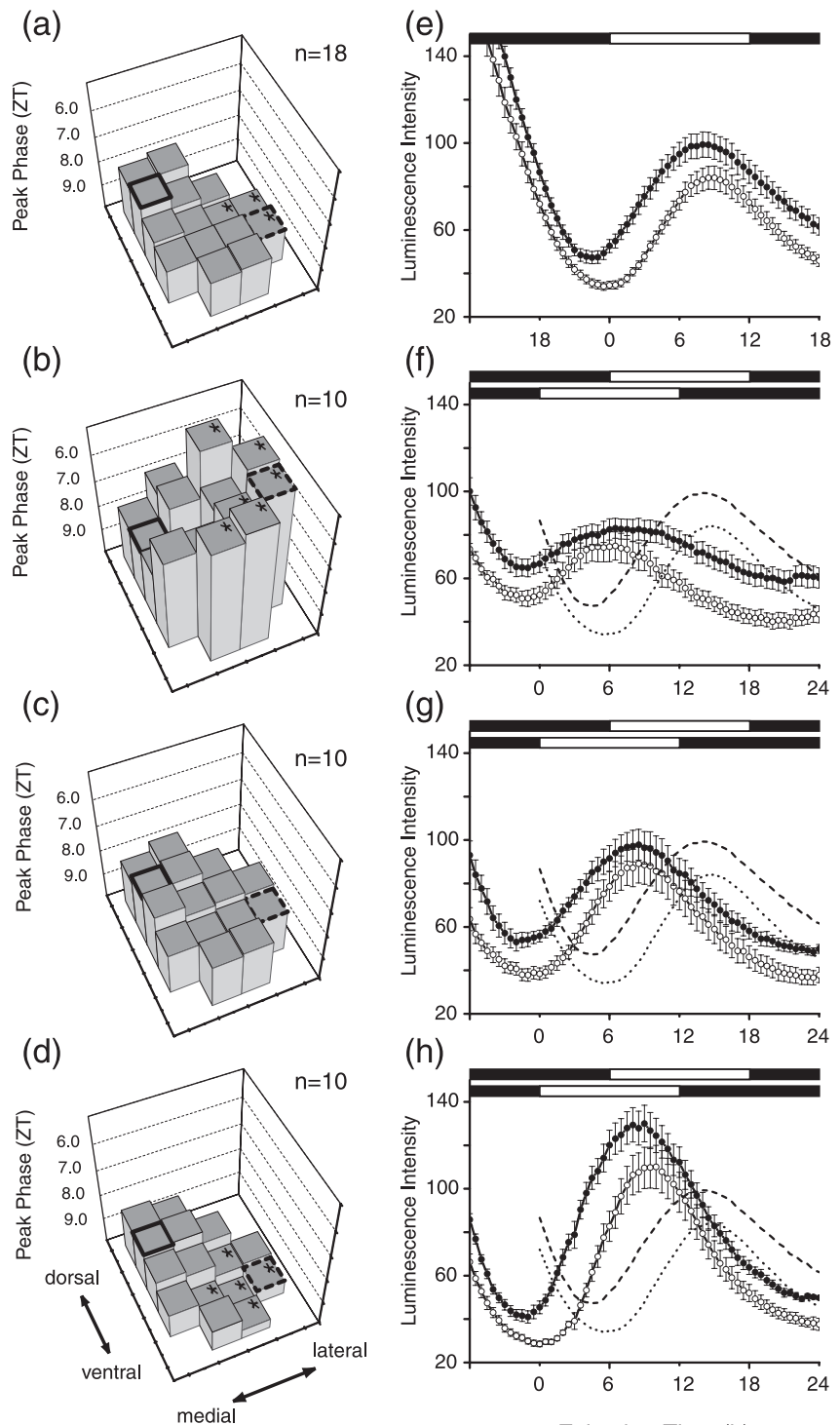

(h)

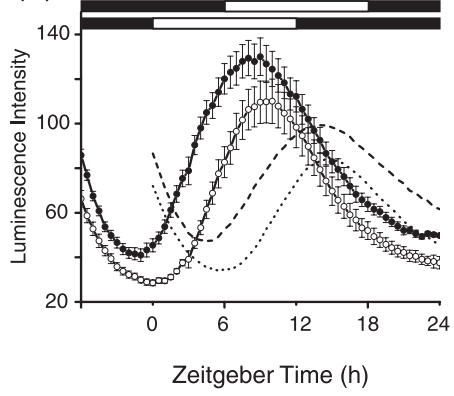

Figure 4. Reentrainment of Per1 expression in the $\mathrm{SCN}$ to $6 \mathrm{~h}$ phase advance in the light cycle. $\boldsymbol{a}$, Control phase plane. $\boldsymbol{b}$, Phase plane on day 1 after $6 \mathrm{~h}$ advance in light schedule. The orientation of the phase plane formed by the tops of the histograms reveals that the lateral region of the SCN has shifted significantly more than the medial regions. c, On day 3 after the light schedule change, the phase relationships have not returned to normal, as indicated by the lack of a phase difference between the dorsomedial and ventrolateral regions. $\boldsymbol{d}$, By $6 \mathrm{~d}$ after the advance in the light schedule, the regional phase differences have been restored.e, Per1-luc rhythms in dorsomedial and ventrolateral regions of the SCN before shift in light schedule (control). $\boldsymbol{f}-\boldsymbol{h}$, Per1-luc rhythms on days 1,3 , and 6 after light cycle advance. In $\boldsymbol{e}-\boldsymbol{h}$, error bars indicate SE. The conventions are the same as in Figure 3.

\section{Discussion}

There is increasing evidence from several rodent species that the SCN exhibits regionally specific characteristics that may underlay specialized functions in the rhythmic control of physiology and behavior (Yan et al., 1999; Schwartz et al., 2000; Hamada et al., 2001, 2004; Moore et al., 2002; Nagano et al., 2003; Schaap et al., 2003; Yamaguchi et al., 2003; de la Iglesia et al., 2004). Our results, using real-time image analysis, indicate that there are stable phase differences in Perl expression in rat, with phase varying in a topologically specific manner. This observation supports the findings of Yan and Okamura (2002), who, using in situ hybridization techniques, reported a gradient in Per1 expression, in vivo, 
beginning in the dorsomedial SCN and then spreading laterally. The results are also consistent with experiments on the SCN of mouse in which systematic regional differences in phase were detected (Yamaguchi et al., 2003). Although the mechanism of the gradient expression is not clear, our result indicate that SCN temporal organization is conserved in the isolated SCN tissue slice, because we are able to measure these phase differences when Per1 rhythms are recorded in vitro.

Our results confirm previous studies (Yamazaki et al., 2000) that the Per1 rhythm measured over the entire SCN shifts rapidly in response to delays or advances in the light schedule. It differs slightly from the previous study in that the Perl-luc steady-state rhythm was more delayed (ZT $8.4 \mathrm{~h}$ vs ZT 6.9). It also differs from the previous work in the observation of an overshoot of the phase advance on the first cycle after the shift in the light schedule [the Yamazaki et al. (2000) study did not evaluate the third day after the light schedule advance]. We are uncertain about the basis of these differences, which could be attributable to the different rat line used in this study [W(perl)2 vs W(perl)1], the use of homozygotes versus heterozygotes, slightly different sized tissue slices, the culture medium, or, less likely, the measurement technology (video imaging of SCN vs photometry).

One possible source of experimental error that could affect the magnitude of the steady-state phase shifts in our experiments would be a change in the circadian time of dissection, in preparations in which the phase shift was still incomplete. If the procedure itself were to generate phase shifts that differed depending on dissection time, the ultimate phase of the incompletely shifted SCN might be differentially affected by the dissection procedure. We think it is unlikely that any underlying phase-response curve to dissection would have played a major role in determining the phase of the SCN in our experiments. In the case of phase advances, in which there is evidence that the behavior does shift slowly, an incomplete phase shift would have caused dissection time to move to early circadian times, toward the middle of the subjective day. We have not observed an effect on phase of dissections during the middle of the day compared with the end of the day (T. Yoshikawa, S. Yamazaki, and M. Menaker, unpublished observations). Consequently, we do not suspect that dissection time is an important factor in our experiments.

Our ability to measure the phase of the Perl-luc rhythm in different regions of the SCN reveals that the SCN undergoes transient desynchronization in Per1 rhythmicity after shifts in the light schedule. The desynchronization arises from different regions of the SCN readjusting at different rates. A similar result has been reported by Nagano et al. (2003) in an elegant study using in situ hybridization. The present study confirms and extends the results of Nagano et al. (2003) in that we are able to show that the phase differences measured in various regions of the SCN persist in the absence of feedback from behavioral activity or modulation from brain regions outside of the SCN and the small surrounding area present in the brain slice. This is a nontrivial issue, insofar as SCN electrical activity is influenced by locomotor behavior and other CNS inputs (Meijer et al., 1997; Yamazaki et al., 1998; Deboer et al., 2003), and consequently, Per1 activity measured in vivo may reflect these extrinsic factors and not reveal the actual state of SCN oscillators.

In addition to changes in interregional phase differences after light schedule shifts, we also observed changes in the amplitude of the luminescence signals. Although in steady state we normally found the dorsomedial region to be brighter than the ventrolateral region, this difference appeared to increase transiently after a phase delay in the light schedule and possibly diminish during a phase advance. We are uncertain about the significance of such amplitude changes. The differences could reflect changes in the coherence of individual oscillators within a region during phase transitions or could be attributable to changes within individual neurons.

The regional differences that we observe in phase-shifting behavior may have their origins in the difference in how light information reaches different regions of the SCN. During the subjective night, light exposure induces Perl expression that is limited to the ventrolateral SCN, with no induction detected in dorsomedial region (Shigeyoshi et al., 1997; Yan et al., 1999). Our findings suggest that after the first cycle of a shifted light schedule, Perl in the ventrolateral SCN is not only induced by light but also shifts more than other regions. This may be related to the fact that the ventrolateral region receives direct retinal input that then must spread to other regions (Romijn et al., 1997). One attractive hypothesis is that the oscillators in the dorsomedial region are coupled to oscillators in the ventrolateral SCN via pathways that have been described previously (Watanabe et al., 2000; Aida et al., 2002). The phase readjustment in the ventrolateral neurons may lead to electrical activity in dorsomedial neurons at times within the cycle when such activity generates phase shifts. Similarly, through bidirectional coupling, the dorsomedial oscillators may act to retard the oscillators in the ventrolateral region, reducing the $9 \mathrm{~h}$ phase advance to the steady-state $6 \mathrm{~h}$ phase shift. The situation with phase advances is perhaps the most unusual. In addition to the overshoot of steady-state phase by the ventrolateral region, after the shift in the light schedule, it appears that the dorsomedial region rapidly makes a complete phase shift, as estimated from peak activity. However, the rhythm amplitude is significantly damped and broadened. Although there are several possible explanations for the change in waveform, one possibility is that phase differences have arisen among oscillator neurons within the dorsomedial region, leading to a loss of rhythm coherence. What is notable and unexpected is that all regions of the SCN exhibit greater phase shifts after the exposure to the advanced light schedule than compared with a delayed schedule; however, it then takes longer to reestablish interregional phase relationships for phase advances (i.e., regaining normal temporal organization). In this regard, our results differ from the previous in vivo study by Nagano et al. (2003). Although they also observed an overshoot in the ventrolateral region after a $6 \mathrm{~h}$ phase advance in the light schedule, they did not observe, as we did, a nearly complete phase advance in the dorsomedial region after one cycle of the shifted schedule. In their study, the dorsomedial region tracked the slow resetting kinetics of the locomotor behavior. These differences in results may reflect the action of feedback from locomotor activity and other oscillators that could act on the SCN in situ.

One important and unresolved issue is the extent to which feedback on the SCN from locomotor activity influences the phase of oscillators within different SCN regions. Locomotor activity acutely decreases SCN electrical activity (Meijer et al., 1997; Yamazaki et al., 1998; Schaap and Meijer, 2001) and Per1 expression (Maywood et al., 1999). Nagano et al. (2003) showed that after a light schedule shift, the Per1 oscillation in the dorsomedial SCN and the locomotor rhythm appeared to follow a similar time course. They noted an inverse relationship between locomotor activity and Per 1 expression in the dorsomedial SCN (Nagano et al., 2003). It is unclear to what extent this phase relationship reflects the dorsomedial region controlling locomotor expression or, alternately, locomotor activity influencing the phase of dorsomedial oscillators. 
The relationship between different SCN regions and locomotor activity can be complex and may reflect the state of synchrony within the SCN. A recent study in which rats were exposed to non-24 h light schedules, leading to two dominant periodicities in the locomotor record, revealed that molecular rhythms in the ventrolateral and dorsomedial SCNs were expressed in antiphase when the rhythms were measured during the subjective night of one rhythmic locomotor component and the subjective day of the other (de la Iglesia et al., 2004). Although one cannot rule out feedback from locomotor activity to the SCN in driving the apparent internal desynchronization, the result raises the intriguing issue that rhythmicity in different regions of the SCN may exert control over different components of the locomotor record. It will be important to confirm this observation under more naturalistic $24 \mathrm{~h}$ entrainment conditions.

During phase shifts, there is evidence for temporal desynchrony at many levels of organization. At the transcriptional level, within the SCN, there is evidence for changes in the phase relationships among genes during phase readjustments brought about by light cycle shifts. Reddy et al. (2002) reported a rapid shift of Per1 and Per2 oscillations and a relatively slow shift of Cryptochrome 1 after an acute LD cycle shift. In addition, Per 1 and Per2 have been found to behave differently during advancing and delaying phase shifts (Albrecht et al., 2001; Yan and Silver, 2002). At the tissue level, there is evidence that Per1 and electrical rhythmicity dissociate during light schedule shifts (Vansteensel et al., 2003). From our study and that by Nagano et al. (2003), there is evidence for regionally specific phase shifting kinetics of circadian rhythms in transcriptional activity. Finally, at the system level, there is a clear indication that different peripheral tissues (Yamazaki et al., 2000) and different brain regions (Abe et al., 2002) exhibit different resetting behavior than the SCN or locomotor behavior. The results from many of these studies suggest that temporal organization within mammals is complex, and resynchronization to new light schedules involves complex adjustments at many levels of organization including within individual SCN pacemaker neurons, within the SCN tissue, and between the SCN and other circadian rhythm generators.

\section{References}

Abe M, Herzog ED, Yamazaki S, Straume M, Tei H, Sakaki Y, Menaker M, Block GD (2002) Circadian rhythms in isolated brain regions. J Neurosci 22:350-356.

Aida R, Moriya T, Araki M, Akiyama M, Wada K, Wada E, Shibata S (2002) Gastrin-releasing peptide mediates photic entrainable signals to dorsal subsets of suprachiasmatic nucleus via induction of Period gene in mice. Mol Pharmacol 61:26-34.

Akiyama M, Kouzu Y, Takahashi S, Wakamatsu H, Moriya T, Maetani M, Watanabe S, Tei H, Sakaki Y, Shibata S (1999) Inhibition of light- or glutamate-induced mPerl expression represses the phase shifts into the mouse circadian locomotor and suprachiasmatic firing rhythms. J Neurosci 19:1115-1121.

Albrecht U, Sun ZS, Eichele G, Lee CC (1997) A differential response of two putative mammalian circadian regulators, mper 1 and mper2, to light. Cell 91:1055-1064.

Albrecht U, Zheng B, Larkin D, Sun ZS, Lee CC (2001) MPer1 and mper2 are essential for normal resetting of the circadian clock. J Biol Rhythms 16:100-104.

Balsalobre A, Damiola F, Schibler U (1998) A serum shock induces circadian gene expression in mammalian tissue culture cells. Cell 93:929-937.

Daan S, Pittendrigh CS (1976) A functional analysis of circadian pacemakers in nocturnal rodents. J Comp Physiol 106:253-266.

Deboer T, Vansteensel M, Detari L, Meijer J (2003) Sleep states alter activity of suprachiasmatic nucleus neurons. Nat Neurosci 6:1086-1090.

de la Iglesia HO, Cambras T, Schwartz WJ, Diez-Noguera A (2004) Forced desynchronization of dual circadian oscillators within the rat suprachiasmatic nucleus. Curr Biol 14:796-800.
Hamada T, LeSauter J, Venuti JM, Silver R (2001) Expression of Period genes: rhythmic and nonrhythmic compartments of the suprachiasmatic nucleus pacemaker. J Neurosci 21:7742-7750.

Hamada T, Antle MC, Silver R (2004) Temporal and spatial expression patterns of canonical clock genes and clock-controlled genes in the suprachiasmatic nucleus. Eur J Neurosci 19:1741-1748.

Hida A, Koike N, Hirose M, Hattori M, Sakaki Y, Tei H (2000) The human and mouse Period 1 genes: five well-conserved E-boxes additively contribute to the enhancement of mPer1 transcription. Genomics 65:224-233.

Johnson RF, Morin LP, Moore RY (1988) Retinohypothalamic projections in the hamster and rat demonstrated using cholera toxin. Brain Res 462:301-312.

Maywood ES, Mrosovsky N, Field MD, Hastings MH (1999) Rapid downregulation of mammalian period genes during behavioral resetting of the circadian clock. Proc Natl Acad Sci USA 96:15211-15216.

Meijer JH, Schaap J, Watanabe K, Albus H (1997) Multiunit activity recordings in the suprachiasmatic nuclei: in vivo versus in vitro models. Brain Res 753:322-327.

Moga MM, Moore RY (1997) Organization of neural inputs to the suprachiasmatic nucleus in the rat. J Comp Neurol 389:508-534.

Moore RY (1973) Retinohypothalamic projection in mammals: a comparative study. Brain Res 49:403-409.

Moore RY, Eichler VB (1972) Loss of a circadian adrenal corticosterone rhythm following suprachiasmatic lesions in the rat. Brain Res 42:201-206.

Moore RY, Speh JC, Leak RK (2002) Suprachiasmatic nucleus organization. Cell Tissue Res 309:89-98.

Muscat L, Huberman AD, Jordan CL, Morin LP (2003) Crossed and uncrossed retinal projections to the hamster circadian system. J Comp Neurol 466:513-524.

Nagano M, Adachi A, Nakahama K, Nakamura T, Tamada M, MeyerBernstein E, Sehgal A, Shigeyoshi Y (2003) An abrupt shift in the day/ night cycle causes desynchrony in the mammalian circadian center. J Neurosci 23:6141-6151.

Nakamura W, Honma S, Shirakawa T, Honma K (2001) Regional pacemakers composed of multiple oscillator neurons in the rat suprachiasmatic nucleus. Eur J Neurosci 14:666-674.

Quintero JE, Kuhlman SJ, McMahon DG (2003) The biological clock nucleus: a multiphasic oscillator network regulated by light. J Neurosci 23:8070-8076.

Ralph MR, Foster RG, Davis FC, Menaker M (1990) Transplanted suprachiasmatic nucleus determines circadian period. Science 247:975-978.

Reddy AB, Field MD, Maywood ES, Hastings MH (2002) Differential resynchronisation of circadian clock gene expression within the suprachiasmatic nuclei of mice subjected to experimental jet lag. J Neurosci 22:7326-7330.

Reppert SM, Weaver DR (2002) Coordination of circadian timing in mammals. Nature 418:935-941.

Romijn HJ, Sluiter AA, Pool CW, Wortel J, Buijs RM (1997) Evidence from confocal fluorescence microscopy for a dense, reciprocal innervation between AVP-, somatostatin-, VIP/PHI-, GRP-, and VIP/PHI/GRPimmunoreactive neurons in the rat suprachiasmatic nucleus. Eur J Neurosci 9:2613-2623.

Schaap J, Meijer JH (2001) Opposing effects of behavioural activity and light on neurons of the suprachiasmatic nucleus. Eur J Neurosci 13:1955-1962.

Schaap J, Albus H, VanderLeest HT, Eilers PH, Detari L, Meijer JH (2003) Heterogeneity of rhythmic suprachiasmatic nucleus neurons: implications for circadian waveform and photoperiodic encoding. Proc Natl Acad Sci USA 100:15994-15999.

Schwartz WJ, Carpino Jr A, de la Iglesia HO, Baler R, Klein DC, Nakabeppu Y, Aronin N (2000) Differential regulation of fos family genes in the ventrolateral and dorsomedial subdivisions of the rat suprachiasmatic nucleus. Neuroscience 98:535-547.

Shearman LP, Zylka MJ, Weaver DR, Kolakowski Jr LF, Reppert SM (1997) Two period homologs: circadian expression and photic regulation in the suprachiasmatic nuclei. Neuron 19:1261-1269.

Shigeyoshi Y, Taguchi K, Yamamoto S, Takekida S, Yan L, Tei H, Moriya T, Shibata S, Loros JJ, Dunlap JC, Okamura H (1997) Light-induced resetting of a mammalian circadian clock is associated with rapid induction of the mPer1 transcript. Cell 91:1043-1053.

Stephan FK, Zucker I (1972) Circadian rhythms in drinking behavior and 
locomotor activity of rats are eliminated by hypothalamic lesions. Proc Natl Acad Sci USA 69:1583-1586.

Stokkan KA, Yamazaki S, Tei H, Sakaki Y, Menaker M (2001) Entrainment of the circadian clock in the liver by feeding. Science 291:490-493.

Tei H, Okamura H, Shigeyoshi Y, Fukuhara C, Ozawa R, Hirose M, Sakaki Y (1997) Circadian oscillation of a mammalian homologue of the Drosophila period gene. Nature 389:512-516.

Tischkau SA, Mitchell JW, Tyan SH, Buchanan GF, Gillette MU (2003) $\mathrm{Ca}^{2+} / \mathrm{cAMP}$ response element-binding protein (CREB)-dependent activation of Per1 is required for light-induced signaling in the suprachiasmatic nucleus circadian clock. J Biol Chem 278:718-723.

Vansteensel MJ, Yamazaki S, Albus H, Deboer T, Block GD, Meijer JH (2003) Dissociation between circadian Perl and neuronal and behavioral rhythms following a shifted environmental cycle. Curr Biol 13:1538-1542.

Watanabe K, Vanecek J, Yamaoka S (2000) In vitro entrainment of the circadian rhythm of vasopressin-releasing cells in suprachiasmatic nucleus by vasoactive intestinal polypeptide. Brain Res 877:361-366.

Yamaguchi S, Isejima H, Matsuo T, Okura R, Yagita K, Kobayashi M, Okamura H (2003) Synchronization of cellular clocks in the suprachiasmatic nucleus. Science 302:1408-1412.
Yamazaki S, Kerbeshian MC, Hocker CG, Block GD, Menaker M (1998) Rhythmic properties of the hamster suprachiasmatic nucleus in vivo. J Neurosci 18:10709-10723.

Yamazaki S, Numano R, Abe M, Hida A, Takahashi R, Ueda M, Block GD, Sakaki Y, Menaker M, Tei H (2000) Resetting central and peripheral circadian oscillators in transgenic rats. Science 288:682-685.

Yan L, Okamura H (2002) Gradients in the circadian expression of Perl and Per2 genes in the rat suprachiasmatic nucleus. Eur J Neurosci 15:1153-1162.

Yan L, Silver R (2002) Differential induction and localization of mPerl and $\mathrm{mPer} 2$ during advancing and delaying phase shifts. Eur J Neurosci 16:1531-1540.

Yan L, Takekida S, Shigeyoshi Y, Okamura H (1999) Perl and Per2 gene expression in the rat suprachiasmatic nucleus: circadian profile and the compartment-specific response to light. Neuroscience 94:141-150.

Yoo SH, Yamazaki S, Lowrey PL, Shimomura K, Ko CH, Buhr ED, Siepka SM, Hong HK, Oh WJ, Yoo OJ, Menaker M, Takahashi JS (2004) PERIOD2::LUCIFERASE real-time reporting of circadian dynamics reveals persistent circadian oscillations in mouse peripheral tissues. Proc Natl Acad Sci USA 101:5339-5346. 